stores in the nervous system, where stores have usually been thought of as slower and modulatory. For example, glutamate release from parallel fibers activates metabotropic glutamate receptors on Purkinje cell dendritic spines, which in turn trigger a large postsynaptic release from IP3-sensitive calcium stores $^{8,9}$. The time course of this release is on the order of hundreds of milliseconds. At some presynaptic terminals, calcium stores are involved in controlling resting and residual calcium due to posttetanic potentiation, which takes place over tens of seconds ${ }^{10}$. By contrast, the present study by Llano and colleagues suggests that calcium stores can synchronize release to within a few milliseconds (Fig. 1).

This ability to synchronize release on short time scales raises the possibility that stores contribute to fast neurotransmitter release, also called phasic release. Previously, the only source of the high concentrations of calcium that drive phasic release was believed to be voltage-gated calcium channels, which are tightly coupled to the synaptic release machinery ${ }^{11}$. Recently, calcium-permeable ionotropic receptors have been implicated as additional potential sources of calcium. For example, calcium influx through NMDA receptors alone can trigger GABA release at dendrodendritic synapses in the olfactory bulb ${ }^{12,13}$. The study by Llano and colleagues indicates that calcium stores can also supply sufficient calcium to drive fast multivesicular release. It is likely, therefore, that the stores lie very close to release sites (within a few hundred nanometers), and it will be interesting to examine this issue through studies of ultrastructure and of exogenous calcium chelators.

There are several interesting questions raised by this work. Mechanistically, how are stores being triggered to release calcium? How many release sites along the basket cell axon are recruited by calcium release from stores? Physiologically, what is the role of calcium stores in transmitter release during normal activity? Are the spontaneous release events themselves important, or do they reflect a process whose primary function is to amplify phasic release? If the latter, can the amplification, and thus synaptic strength, be modulated by activity or chemical messengers? Finally, do other synapses have calcium stores that can drive fast neurotransmitter release? Such large-amplitude mPSCs have not been commonly described in the nervous system, but because the antibody that Llano and colleagues introduced recognizes a previously unknown population of calcium stores, it remains to be seen whether these stores show up in other neuronal types.

1. Llano, I. et al. Nat. Neurosci. 3, 1256-1265 (2000).

2. Tse, F. W., Tse, A., Hille, B., Horstmann, H. \& Almers, W. Neuron 18, 121-132 (1997).

3. Penner, R. \& Neher, E. J. Exp. Biol. 139, 329-345 (1988).

4. Kuba, K. Jpn. J. Physiol. 44, 613-650 (1994).

5. Narita, K. et al. J. Gen. Physiol. 112, 593-609 (1998).

6. Savic, N. \& Sciancalepore, M. Eur. J. Neurosci. 10, 3379-3386 (1998).

7. Niggli, E. Annu. Rev. Physiol. 61, 311-335 (1999).

8. Finch, E. A. \& Augustine, G. J. Nature 396, 753-756 (1998).

9. Takechi, H., Eilers, J. \& Konnerth, A. Nature 396, 757-760 (1998).

10. Tang, Y.-G. \& Zucker, R. S. Neuron 18, 483-491 (1997).

11. Zucker, R. S. J. Physiol. (Paris) 87, 25-36 (1993).

12. Chen, W. R., Xiong, W. \& Shepherd, G. M. Neuron 25, 625-633 (2000).

13. Halabisky, B., Friedman, D., Radojicic, M. \& Strowbridge, B. W. J. Neurosci. 20, 5124-5134 (2000).

\title{
Dissecting the ins and outs of excitement: glutamate receptors on the move
}

\author{
Volker Haucke
}

\section{AMPA, NMDA or insulin can cause endocytosis of AMPA receptors. Two papers now show that these stimuli act via distinct signaling pathways, some of which also induce LTD.}

Ionotropic glutamate receptors (AMPARs) can move into and out of the postsynaptic membrane dynamically. Presumably such changes in the number of AMPARs at the membrane are important in activity-dependent synaptic plasticity, and thus the signaling pathways governing glutamate receptor trafficking have moved to center stage in molecular neurobiology. In this issue, Beattie et al. ${ }^{1}$ and Lin et al. ${ }^{2}$ begin to shed light on the mechanisms by

The author is at the Zentrum für Biochemie \& Molekulare Zellbiologie, Georg-August-

Universität Göttingen, Humboldtallee 23,

D-37073 Göttingen, Germany.

e-mail:vhaucke@gwdg.de which different effectors can induce AMPAR internalization. They find striking similarities between the signaling mechanisms governing AMPAR endocytosis and the induction of long-term depression (LTD). Although some of the results seem to be at odds with each other, these new studies provide a glimpse into an increasingly complex web of signaling networks at glutamatergic synapses.

Because activity-dependent changes in synaptic efficacy correlate with changes in the surface levels of AMPARs, postsynaptic glutamate receptor trafficking between the plasma membrane and internal compartments is proposed to regulate the strength of excitatory synapses. Endocy- totic internalization of most plasma membrane receptors involves clathrin and dynamin, which mediate selective transport from the plasma membrane to internal endosomal compartments. Over the past two years, it has become clear that clathrin-mediated endocytosis is also required for hippocampal long-term depression ${ }^{3-6}$, with AMPARs being internalized rapidly into clathrin-coated vesicles $^{5}$. Conversely, induction of long-term potentiation is accompanied by insertion of AMPARs from intracellular sites into the postsynaptic density ${ }^{7,8}$, presumably by exocytotic membrane fusion. Thus, a picture emerges in which activity influences cycling of ionotropic glutamate receptors ${ }^{9}$ in and out of the membrane.

So which signaling cascades control these processes, and thus synaptic responsiveness? Some answers to this intriguing question have now emerged from two elegant papers by Malenka ${ }^{1}$, Sheng ${ }^{2}$ and their colleagues. By using state-of-the-art immunofluorescence imaging techniques in live cultured hippocampal neurons labeled with antibodies, along with drugs that selectively stimulate distinct pathways of glutamate signaling, the authors provide a nascent picture of how activity may regulate synaptic strength at excitatory 
synapses. Lin et al. ${ }^{2}$ set out to characterize AMPAR internalization in response to different stimuli, such as glutamate receptor ligands, synaptic activity and insulin. They find that agonists that selectively activate AMPA- but not NMDA-type ionotropic glutamate receptors stimulate the basal rate of dynamin-mediated AMPAR internalization via a signaling mechanism involving L-type calcium channels (Fig. 1a). The AMPA receptor blocker CNQX surprisingly also stimulates AMPAR endocytosis, presumably by inducing a conformational change that may facilitate receptor incorporation into clathrin-coated vesicles, but does not activate L-type calcium channels. Thus, one pathway by which synaptically released glutamate may trigger AMPAR internalization is by inducing a ligand-dependent conformational change within the activated open receptor that cooperates with calcium influx through L-type calcium channels to facilitate receptor endocytosis.

In a similar set of experiments, Beattie et al. ${ }^{1}$ describe the effects of both AMPAR and NMDA receptor (NMDAR) activation on AMPAR endocytosis. Treating neuronal cultures with either AMPA or NMDA causes significant calciumdependent AMPAR internalization, but via different signaling cascades. As discussed above, AMPA-induced AMPAR endocytosis requires calcium influx through L-type calcium channels. By contrast, NMDA treatment (Fig. 1b) causes calcium influx through the NMDAR itself, followed by AMPAR endocytosis via the calcium/calmodulin-dependent phosphatase calcineurin, a known regulator of synaptic endocytosis ${ }^{10,11}$. These data may explain the long-standing observation that calcineurin is essential for hippocampal LTD ${ }^{12}$. Although activation of either the AMPA or the NMDA pathway results in the internalization of surface AMPAR to endosomes, NMDA seems to preferentially act in the distal portion of dendrites, suggesting that dendritic and thus presumably synaptic NMDARs are particularly effective at triggering AMPAR internalization. It is therefore possible that different modes of AMPAR internalization are being used in different regions of the neuron.

As often in a rapidly evolving field, some of the results reported by the two groups are hard to reconcile with each other. For example, according to Beattie et al. ${ }^{1}$, AMPA-dependent glutamate receptor endocytosis is blocked by cyclosporin $\mathrm{A}$, an inhibitor of calcineurin, whereas Lin et al. ${ }^{2}$ observe no such effect under similar conditions. One possibility is that these differences reflect the existence of two populations of AMPA receptors on the cell surface, one of which is more stable than the other. Both groups suggest that the protocol used by Beattie et al. ${ }^{1}$ may measure the activity-dependent mode of AMPA-stimulated internalization, subtracting off constitutive endocytosis, whereas the protocol of Lin et al. ${ }^{2}$ may preferentially measure the calcineurin-independent pathway. Resolving these discrepancies clearly will require a detailed analysis with a variety of pharmacological inhibitors and better defined experimental systems that allow a more direct way of dissecting intracellular signal transduction pathways.
Because insulin can stimulate AMPAR internalization that mutually occludes $\mathrm{LTD}^{4}$, insulin treatment was thought to initiate a signaling cascade similar to the one elicited by NMDA. The new studies ${ }^{1,2}$ show that this is only partially true. Insulin seems to trigger AMPAR endocytosis via a distinct pathway that does not require calcium influx ${ }^{1}$, yet depends on clathrin and dynamin ${ }^{2}$. AMPARs internalized in response to insulin show an extremely diffuse staining pattern by immunofluorescence $^{1}$ that differs markedly from the punctate localization of AMPARs internalized in response to AMPA or NMDA ${ }^{1,2}$. Moreover, the internalized receptors enter a distinct membrane compartment-possibly a late endosome-after prolonged chase times $^{2}$ (Fig. 1c), suggesting that they

Fig. 1. Mechanisms of agonist-stimulated ionotropic glutamate receptor internalization. AMPA receptors (red) become sequestered into endocytotic clathrin-coated pits, followed by internalization into endosomal compartments. D ifferent agonists activate distinct signaling pathways that regulate AMPAR internalization and hence the strength of central excitatory synapses. (a) AMPAR activation induces a conformational change within the receptor that leads to calcium influx through voltage-gated calcium channels (blue) and AMPAR endocytosis. This pathway is blocked by nimodipine, an inhibitor of L-type calcium channels. CN Q X, a competitive inhibitor of AMPARs, also stimulates receptor endocytosis to some extent, but does not activate calcium channels. (b) A second mode of activating AMPAR endocytosis and inducing synaptic depression involves calcium influx through activated N MDARs (yellow) and the calcium-dependent protein phosphatase calcineurin. The N MDAR blocker APV or calcineurin inhibitors such as cyclosporin A (C SA) and FK506 selectively inhibit N MDA-induced AMPAR sequestration. Both pathways result in the transport of internalized receptors to endosomes, which may then recycle back to the postsynaptic plasma membrane. (c) Insulin can also promote AMPAR endocytosis by a poorly defined pathway, which may involve both protein kinases and phosphatases.

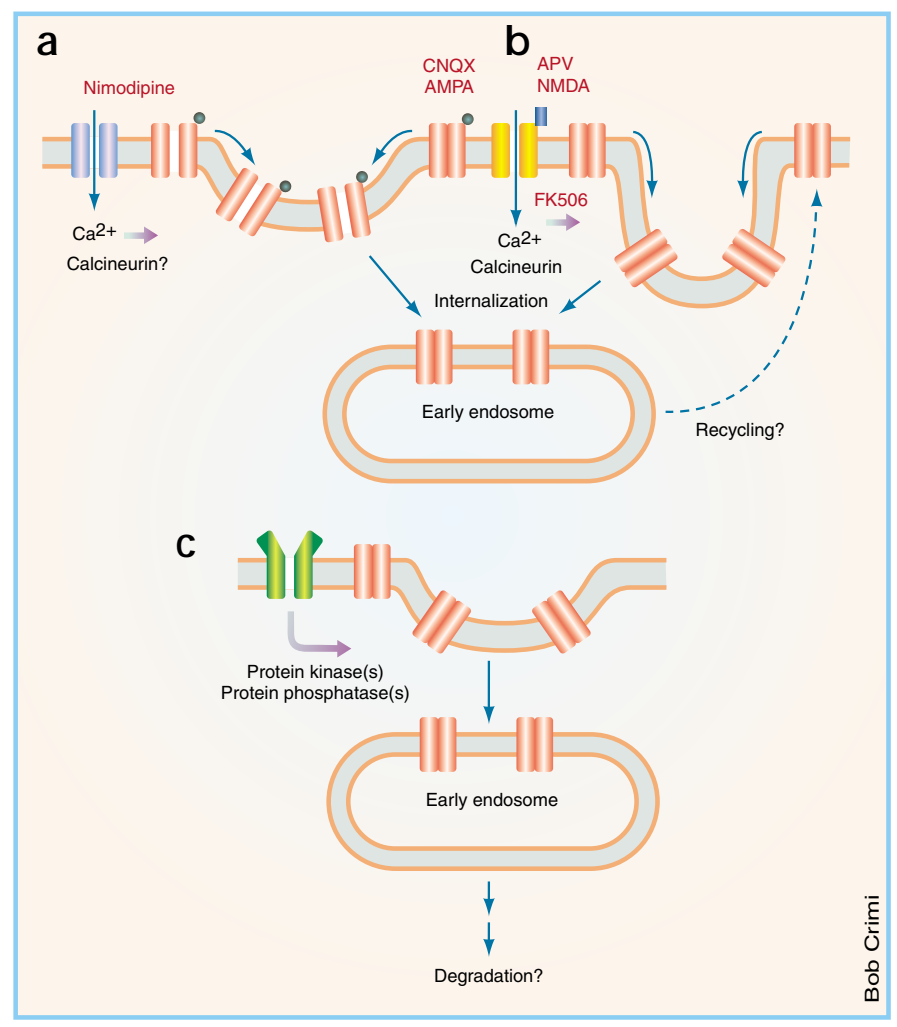


might not be able to recycle back to the plasma membrane.

How the insulin pathway is regulated remains unclear, as both protein kinase ${ }^{1}$ and phosphatase ${ }^{2}$ inhibitors seem to inhibit insulin-mediated glutamate receptor internalization. One possible explanation could be that cycles of phosphorylation and dephosphorylation are involved in the insulin pathway or that multiple components mediate internalization, of which some must be phosphorylated and others dephosphorylated for activation. This second possibility seems less likely in view of studies on synaptic vesicle recycling in the presynaptic nerve terminal, where many of the endocytotic components seem to be in the same (dephosphorylated) state ${ }^{13}$. More work is needed to sort out the mechanistic differences and fine details of this signaling pathway. The most intriguing observation is the differential subcellular distribution of internalized receptors depending on the stimulus. Insulin-triggered AMPAR endocytosis preferentially occurs in the cell soma and, in contrast to the AMPAR- and NMDAR-mediated pathways, does not seem to lead to recycling of receptors to the surface. It is therefore tempting to speculate that AMPA and NMDA mimic physiological responses regulating synaptic plasticity phenomena such as LTD, which happen on a time scale of minutes, whereas the insulin cascade could cause longterm modulation of the relative rates of exo- and endocytosis via irreversible redistribution of AMPARs to late endosomal compartments $^{14}$.

In spite of all the excitement, many unanswered questions remain: how is spatial regulation achieved between discrete portions of the neuron and how are these pathways interconnected? How exactly is the insulin signal transduced? Do all of these pathways eventually converge or are there specific proteins that confer unique properties onto a given pathway? Given the rapid pace of progress in this field, it is likely that we will not have to wait long before the answers to these questions emerge.
1. Beattie, E. C. et al. Nat. Neurosci. 3, 1291-1300 (2000).

2. Lin, J. W. et al. Nat. Neurosci. 3, 1282-1290 (2000).

3. Carroll, R. C., Lissin, D. V., von Zastrow, M., Nicoll, R. A. \& Malenka, R. C. Nat. Neurosci. 2, 454-460 (1999).

4. Carroll, R. C. et al. Proc. Natl. Acad. Sci. USA 96, 14112-14117 (1999).

5. Man, Y. H. et al. Neuron 25, 649-662 (2000).

6. Wang, Y. T. \& Linden, D. J. Neuron 25, 635-647 (2000).

7. Hayashi, Y. et al. Science 287, 2262-2267 (2000).

8. Lledo, P. M., Zhang, X., Sudhof, T. C., Malenka, R. C. \& Nicoll, R. A. Science 279, 399-403 (1998).

9. Turrigiano, G. G. Neuron 26, 5-8 (2000).

10. Lai, M. M. et al. J. Biol. Chem. 274, 25963-25966 (1999).

11. Marks, B. \& McMahon, H. T. Curr. Biol. 8, 740-749 (1998).

12. Mulkey, R. M., Endo, S., Shenolikar, S. \& Malenka, R. C. Nature 369, 486-488 (1994).

13. Slepnev, V. I., Ochoa, G. C., Butler, M. H., Grabs, D. \& De Camilli, P. Science 281, 821-824 (1998).

14. Turrigiano, G. G. \& Nelson, S. B. Curr. Opin. Neurobiol. 10, 358-364 (2000).

analysis of which defined a dozen genes specifically needed for body touch sensation. Two genes from this screen encode channel subunits MEC-4 and MEC-10, which are related to the epithelial amiloride-sensitive sodium channel $(\mathrm{ENaC})$ superfamily $^{6}$ and are expressed nearly exclusively in touch receptor neurons. The nematode channel subunits were named 'degenerins' (DEG) because channel-hyperactivating amino-acid substitutions can induce neuronal degeneration. Importantly, additional channel subunits of the 21-member C. elegans degenerin family have been implicated in mechanical signaling involving other sensory neurons. At least two degenerins are required for nose-touch sensation mediated by head mechanosensory neurons (our unpublished observations), and the UNC-8 degenerin is required for normal locomotion, which seems to depend on stretchsensitive neuronal signaling, analogous to proprioception ${ }^{7}$.

How might degenerin channels sense and transduce mechanical stimuli? The best-developed model is for the MEC-4/MEC-10 touch receptor channel complex $^{2,8}$ (Fig. 1). MEC-4 and MEC-10 are postulated to form a heteromultimeric channel in which extracellular domains interact with proteins situated in a specialized extracellular matrix that encircles the touch receptor neurons. Likewise, the channel intracellular domains may be tied 